\title{
Concept de Modèle Ecologique pour la Zone Humide Alaotra
}

\author{
Hery Lisy T. Ranarijaona
}

\author{
Environnement et BioHydrosystème (EBHS) \\ Valorisation de la Biodiversité Végétale (VBV) \\ Faculté des Sciences Mahajanga \\ Université de Mahajanga Madagascar \\ E-mail: herylisy-simon@moov.mg
}

\begin{abstract}
The wet zone Alaotra consists of the largest lake of Madagascar with about 20,000 ha of open-water and up to 23,000 ha of marshes. This wet zone is unique by the presence of an endemic fauna, and its marshes consist of a monospecific vegetation. The local communities directly depend on this wet zone with fishing and agriculture being an integral part of the region's economy. consequently, various anthropological pressures such as burning of the vegetation, over-fishing, pollution of the lake by the use of weed-killers, and sedimentation of the watersheds due to heavy erosions of the surrounding hills, negatively impact on this ecosystem. The wet zone Alaotra has been classified as an official Ramsar wetland site since 2003 and also as a SAPM protected area in January 2007 because of its ecological importance and its various threats to this ecosystem.

The objective of this article is to present and describe the ecological model, which consists of the identification of the various ecological entities as well as their interdependence, in order to justify the wetlands classification as both a Ramsar and a New Protected Area site. The model has been adopted from Ogden (2005), and its ecological entities integrated in the conceptual model are derived from various research works conducted on the wet zone Alaotra. The model will be critically important in convincing the local authorities as well as the local communities to work together with the researchers, in order to implement the best conservation practices and ensure the sustainable management of the wet zone Alaotra.
\end{abstract}

\section{RESUME}

La zone humide d'Alaotra comprend le plus grand lac de Madagascar qui a une superficie de 20,000 ha ainsi qu'un marais d'une superficie de 23,000 ha. Elle est unique au monde par I'existence d'une faune endémique dont I'habitat est un marais composé d'une végétation mono-spécifique. La vie de la population dépend de cette zone humide, si on ne prend en compte que la pêche, l'agriculture, et l'économie de la région. Cependant, différentes pressions anthropiques affectent cet écosystème telles que la mise à feu du marais et la surpêche, la pollution du lac par l'utilisation des herbicides et la sédimentation à cause d'une forte érosion des bassins versants. Ces pressions causent des impacts sur la faune et la flore de la zone humide d'Alaotra. Cela entraîne un changement environnemental de l'Alaotra, comme la fragmentation du marais, constatée depuis plus de dix ans, et un danger pour la vie de la population aussi bien dans le présent que dans le futur. L'importance de cette zone humide ainsi que les différentes menaces dont elle est la cible, ont conduit a sa classification en tant que site Ramsar en 2003 et fait partie du Système Aire Protégée en janvier 2007.

Cet article a pour objectif de décrire le concept de modèle écologique, qui consiste à identifier les différentes entités écologiques ainsi que leur interdépendance, afin de justifier son classement dans le site Ramsar et en Nouvelle Aire Protégée. Ces entités résultent des différents travaux de recherche effectués sur la zone humide Alaotra. Le modèle adopté est celui d'Ogden (2005). Le modèle écologique présent est une de clé principales de la restauration qui devrait permettre de convaincre les autorités locales ainsi que les villageois à assurer ensemble avec les chercheurs la meilleure conservation et la gestion durable de la zone humide Alaotra.

\section{GENERALITES}

La zone humide Alaotra est située à $170 \mathrm{~km}$ au NE de la capitale de Madagascar. C'est une zone très productive car elle est le premier grenier rizicole du pays. Elle est composée d'un lac et d'un marais. C'est une zone humide unique dans le monde, en raison de la faune endémique unique dont I'habitat est un immense marais. Les principales espèces végétales caractéristiques sont cyperus madagascariensis, cyperus latifolius, abritant des espèces de faune endémique telles que Hapalemur alaotrensis, Microcebus rufus, Salanoia sp. 72 espèces d'oiseaux dépendent de cette zone humide comme habitat permanent ou saisonnier (Pidgeon 1996). La zone humide Alaotra abrite 50 oiseaux en permanence, dont huit endémiques Malagasy (Langrand 1995). 11 espèces de poissons y ont été recensées dont six endémiques et une endémique de la région Alaotra. II s'agit du Rheocles alaotrensis (Bedotidae) (Pidgeon 1996). La zone humide Alaotra souffre de différentes pressions humaines. Deux espèces d'oiseaux endémiques sont en extinction (Tachybaptus rufolavatus et Aythya innotata). De nombreuses recherches ont été entreprises sur la diversité et l'écologie de la faune et de la flore Alaotra. Pour freiner la chasse aux lémuriens et aux oiseaux: I'éducation environnementale ainsi que la sensibilisation ont été employées dans la région (distribution de posters, des articles, du T-shirts sur l'environnement) (Pidgeon 1996). En 2001, une coopération entre les villageois et les autorités 
locales existait pour la préservation du marais, mais sans application des lois. "Si les autorités locales sont sincères sur l'application des Iois, la conservation est induite à Alaotra » (Mutschler 2003). Un contrat renouvelable de trois ans a été accordé par l'état, aux villageois aux alentours de la zone humide Alaotra. Ce contrat était pour objet d'un transfert local de la gestion de la zone humide (plus de $35 \%$ de la superficie du marais). Un programme de sensibilisation et éducation villageoise a été engagée par Durrell Wildlife depuis 2001, dans le but de gérer la zone humide Alaotra de manière durable, et afin de démontrer l'importance de la biodiversité dans la zone humide Alaotra. Par ailleurs, les associations villageoises ont crée le Dina, pour qu'il y ait une bonne gestion de la zone humide en respectant les lois existantes. Une régénération de la végétation du marais est constatée si on fait une comparaison de son état (en 2005) par rapport à celui de 1997. Cette zone humide est accordée comme étant un site Ramsar en 2003 (Andrianandrasana et al. 2005). En janvier 2007, la zone humide Alaotra est classée officiellement dans le site Ramsar, en Nouvelle Aire Protégée dans le cadre du Système d'Aire Protégée de Madagascar.

Cet article a pour objectif d'établir un concept de modèle écologique pour la zone humide Alaotra. Il s'agit d'un plan qui identifie les pressions anthropogéniques majeures, les impacts, ainsi que les attributs biologiques ou les indicateurs des réponses écologiques d'un milieu (Ogden 2005). Compte tenu du degré d'endémisme local de ladite zone humide et la pression humaine qui y pèse, avec une fragmentation du marais non négligeable constatée depuis longtemps, sa préservation est prioritaire, vu l'importance économique aussi bien I'agriculture que I'aquaculture.

SITE D'ETUDE La zone humide Alaotra est située à une altitude de $750 \mathrm{~m}$, à $170 \mathrm{~km}$ au Nord Est de la capitale (Figure 1) (Minenvef et al. 2005). C'est une zone humide composée d'un lac, qui est le plus grand lac malagasy avec une superficie de 20,000 ha et d'un marais avec une surface de 23,000 ha (Andrianandrasana et al. 2005). Elle est alimentée par trois principales rivières qui sont I'Anony, la Sahamoloto et la Sahabe (Aldegheri 1972).

Selon Ferry et al. (1999), son pH varie de 5.5 à 8.1, et sa conductivité est relativement basse, variant de $45.6 \mu \mathrm{S} / \mathrm{cm}$ à $64.7 \mu \mathrm{S} / \mathrm{cm}$. De 1987 à 1995, la teneur en anions et en cations diminue de $0.575 \mathrm{meq} / \mathrm{l}$ à $0.161 \mathrm{meq} / \mathrm{I}$ tandis que celle de la silice augmente de $0.500 \mathrm{mg} / \mathrm{l}$ à $4.700 \mathrm{mg} / \mathrm{l}$.

La zone humide Alaotra est influencée par deux saisons: la saison sèche et la saison humide. La température moyenne maximale de $28.4^{\circ} \mathrm{C}$ est enregistrée en Janvier tandis que la température moyenne minimale est de $22.8^{\circ} \mathrm{C}$ en Juillet.

\section{METHODOLOGIE}

Elle consiste à (1) rassembler tous les résultats de travaux effectués dans la zone humide Alaotra par la recherche bibliographique d'une part et notre recherche personnelle sur la végétation de la zone humide Alaotra d'autre part; la flore et la faune de la zone humide sont identifiées; l'état de dégradation de l'habitat de la faune endémique est présenté: une fragmentation non négligeable du marais Alaotra constatée depuis plus de dix ans démontrée par la carte de végétation du marais Alaotra (établie en Juillet-Août 1997), permettrait d'avoir une réflexion et une justification de la conservation

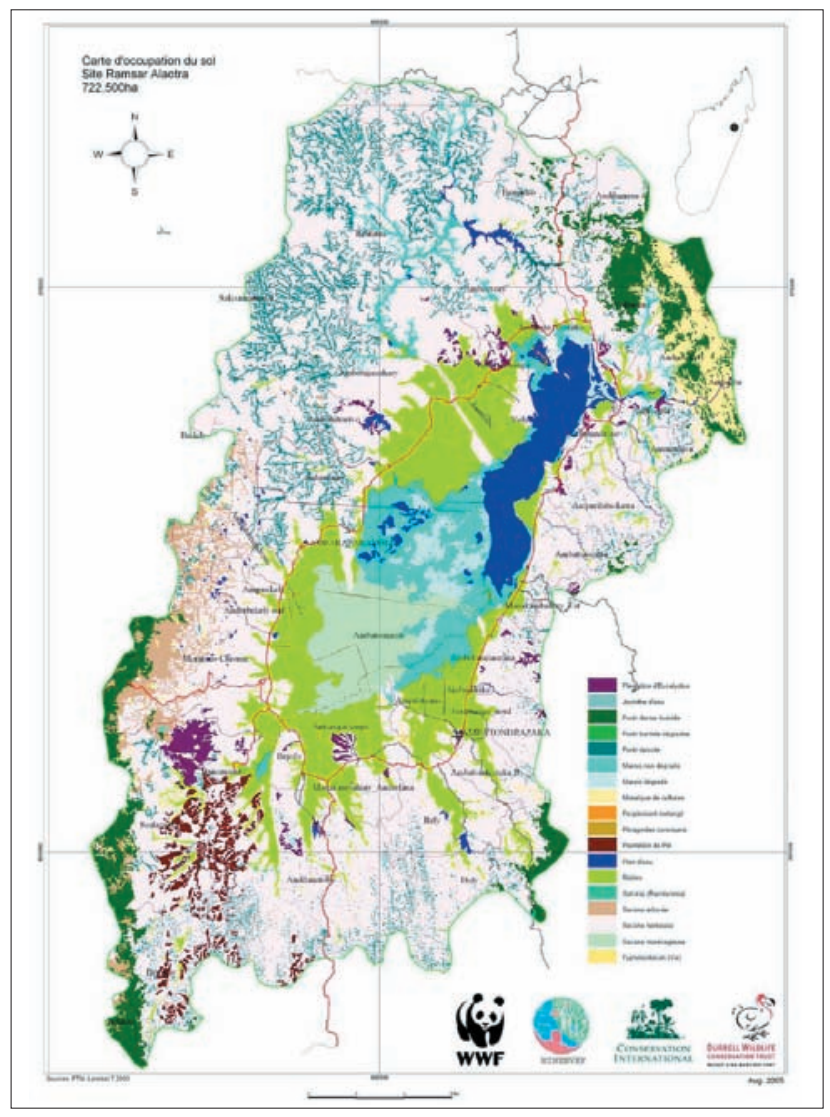

FIGURE 1. La zone humide Alaotra (modifiée d'après la carte d'occupation des sols site Ramsar Alaotra Août 2005, source FTM Landsat 7 2000).

en site Ramsar de la zone humide Alaotra, en comparant cet écosystème à la situation la plus récente; (2) identifier les rôles que peuvent prendre les différentes entités écologiques. Un diagramme est dressé pour montrer leur interdépendance, afin que l'homme soit persuadé de l'importance de cette zone humide. Le modèle adopté est celui d'Ogden (2005). Cette méthode consiste à grouper les attributs.

- Les attributs ou indicateurs sont composés de flore et faune qui participent au fonctionnement de l'écosystème.

- Les influences anthropogéniques sont toutes pressions qui induisent un changement néfaste sur la zone humide Alaotra.

- Les impacts écologiques sont tous changements physiques, chimiques ou biologiques induits par les pressions anthropogéniques.

- Les réponses écologiques sont considérées comme étant toutes réactions causées par les influences anthropogéniques.

Le concept de modèle écologique ainsi que la carte de végétation montrant la fragmentation du marais Alaotra sont des outils pour justifier les intérêts du classement de la zone humide Alaotra dans le site Ramsar (en août 2003). Elle était classée officiellement dans le site Ramsar de Madagascar et en nouvelle aire protégée en janvier 2007.

\section{RESULTATS}

Les résultats suivants présentent le diagramme du concept de modèle écologique pour la zone humide Alaotra (Figure 2). 
LES ATTRIBUTS OU INDICATEURS ECOLOGIQUES

Flore: 35 espèces végétales ont été recensées dans le marais tandis que 52 espèces sont présentes dans le lac Alaotra (Ranarijaona, 1998). Parmi ces plantes, trois sont endémiques, citons Melanthera scandens subsp. madagascariensis, Cynosorchis gracilis et Utricularia perpusilla. Le marais est composé de deux principales espèces qui sont: Cyperus madagascariensis (Photo 1) et Cyperus latifolius, formant chacune un peuplement monospécifique (Figure 3). Typhonodorum lindleyanum (Photo 2) forme également un peuplement mono-spécifique mais avec une superficie beaucoup plus petite par rapport aux deux autres espèces (Figure 3). La zone à Typhonodorum est toujours une zone en eau, dans laquelle il est difficile de mettre le feu par rapport aux deux autres zones. Phragmites mauritianus (Photo 3) est I'espèce la plus commune qui colonise facilement le milieu dégradé plus ou moins asséché. C'est une espèce qui s'adapte plus facilement aussi bien en eau qu'en milieu asséché. Ces espèces servent d'habitat et de nourriture pour la faune; Typhonodorum lindleyanum, Eichhornia crassipes sont mangés par les porcs et les zébus, tandis que Potamogeton octandrus, Ceratophyllum demersum, les Utricularia, rencontrées dans des zones en eau temporaire servent de nourriture pour les oiseaux et les poissons. Cependant, elles sont en compétition avec les espèces envahissantes. D'autres espèces telles que Cyperus madagascariensis, Cyperus latifolius, Phragmites mauritianus, sont utilisées comme matériaux de constructions et artisanaux (fabrication des paniers et de nattes). Les plantes aquatiques sont également importantes pour la population locale par le fait qu'elles sont employées dans la médecine traditionnelle. Au point de vue écologique, Pychnostachys caerulea, Echinochloa stagnina, Aeschynomene elaphroxylon, Salvinia hastata et Eichhornia crassipes prédominent en général dans le lac. Cependant, des espèces envahissantes flottantes telles que Salvinia hastata et Eichhornia crassipes colonisent l'eau libre d'une zone de marais ayant subi la pression humaine; tandis que des espèces herbacées telles que Polygonum wellensi, $P$. tomentosum colonisent les milieux inondés en permanence.

Faune: Poissons: Six espèces sont endémiques dans la zone humide Alaotra. Citons quelques exemples: Paratilapia polleni (Cichlidae), Rheocles alaotrensis (Astherinidae), Ratsirakia legendrei (Eleotridae). Beaucoup d'espèces sont introduites telles que Tilapia zillii, Oreochromis macrochir, o. niloticus et o. mossambicus, Ophiocephalus striatus (Fibata), Gambusia holbrooki, Cyprinus carpio et Carassius auratus. Les tilapia représentent $84.02 \%$ de la population des poissons dans le lac Alaotra. Ils sont très remarqués aussi bien par leur densité que par leur poids moyen (Pidgeon 1996).

Oiseaux: 72 espèces d'oiseau en permanence ou en visite temporaire ont été recensées dans la zone humide Alaotra. 50 espèces sont présentes en permanence dans le lac dont deux en extinction. Les espèces les plus communes sont Anas melleri, A. hottentota, A. erythrorhyncha (Mutschler 2001).

Mammifères: Selon Pidgeon (1996), neuf mammifères ont été identifiés dans le marais Alaotra. Citons les primates tels que l'espèce Hapalemur alaotrensis (endémique de la région), Microcebus rufus, Salanoia concolor; les carnivores qui sont Viverricula indica et Salanoia concolor. Les rongeurs sont Brachyuromys betsileonsis et Rattus rattus, et les insectivores sont Suncus marinus et Microgalus cowani.

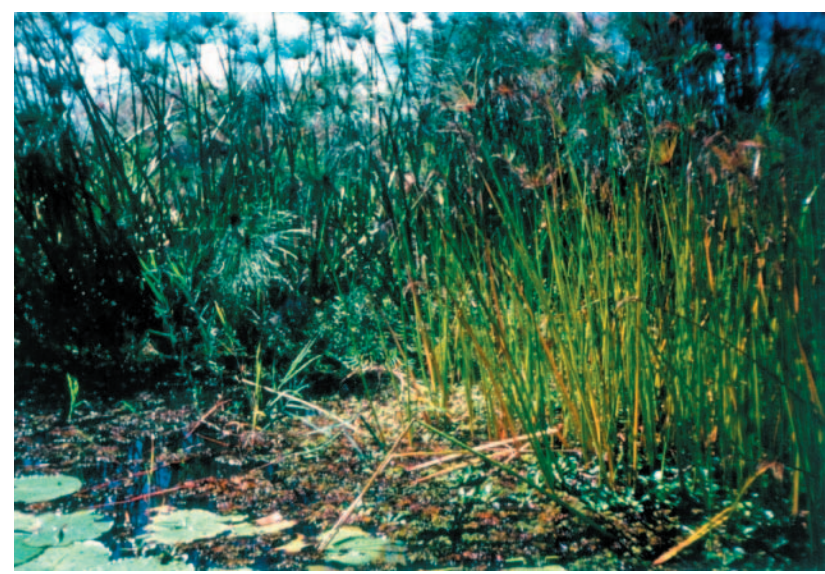

РнОтО 1. Cyperus madagascariensis (Wild.) Roem\&Schult (Cyperaceae)

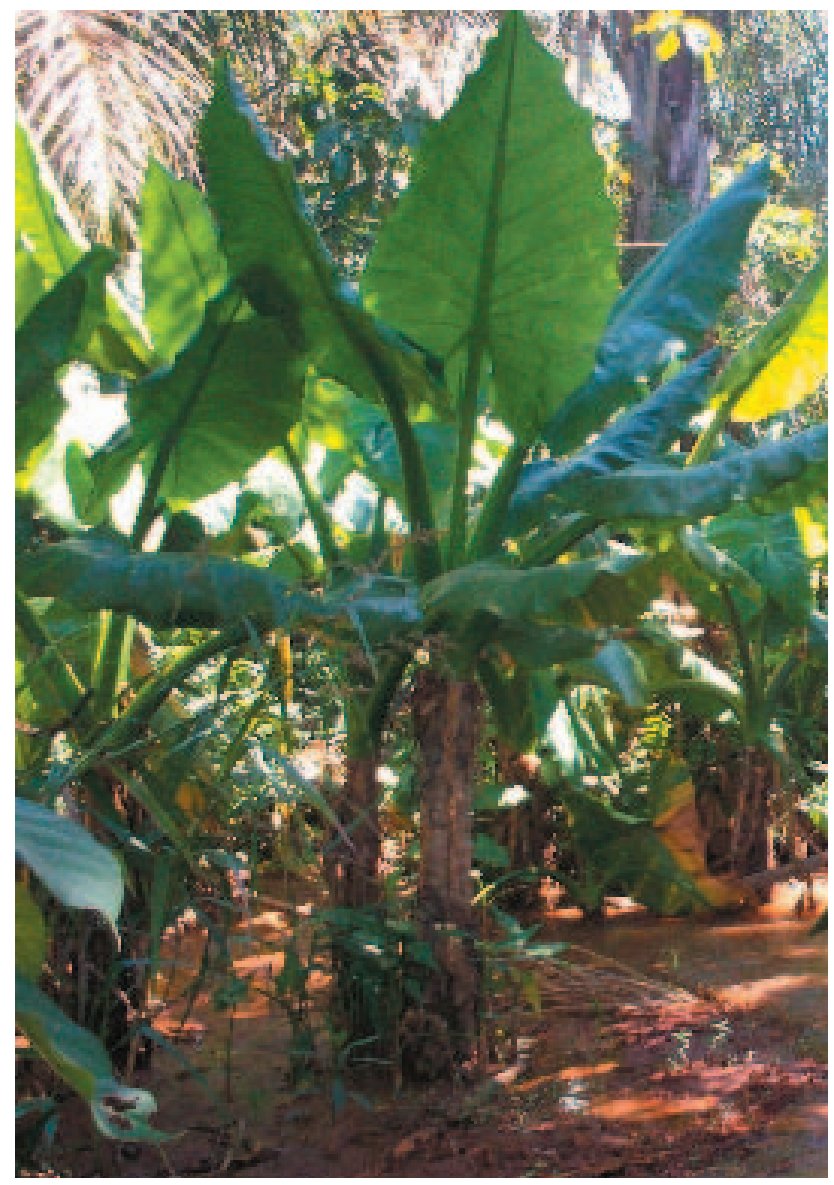

PHOTO 2. Typhonodorum lindleyanum Schott (Araceae)

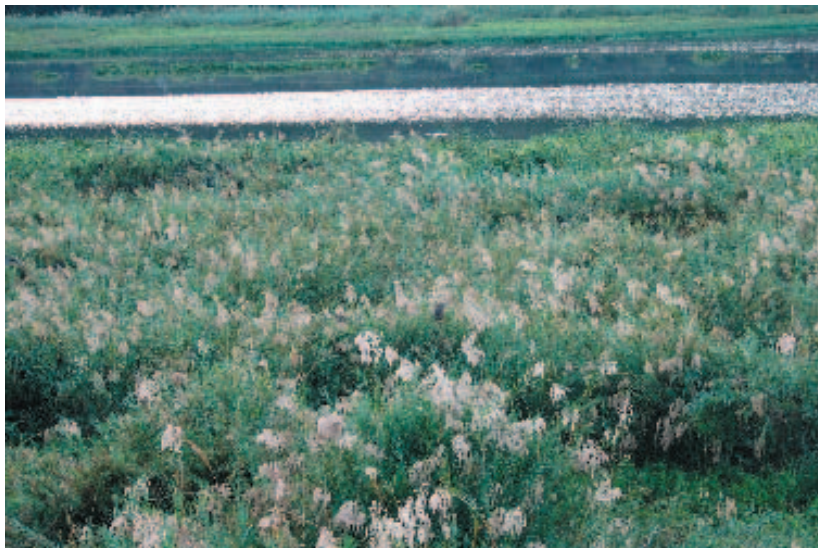

PHOTO 3. Phragmites mauritianus Kunth (Poaceae) 


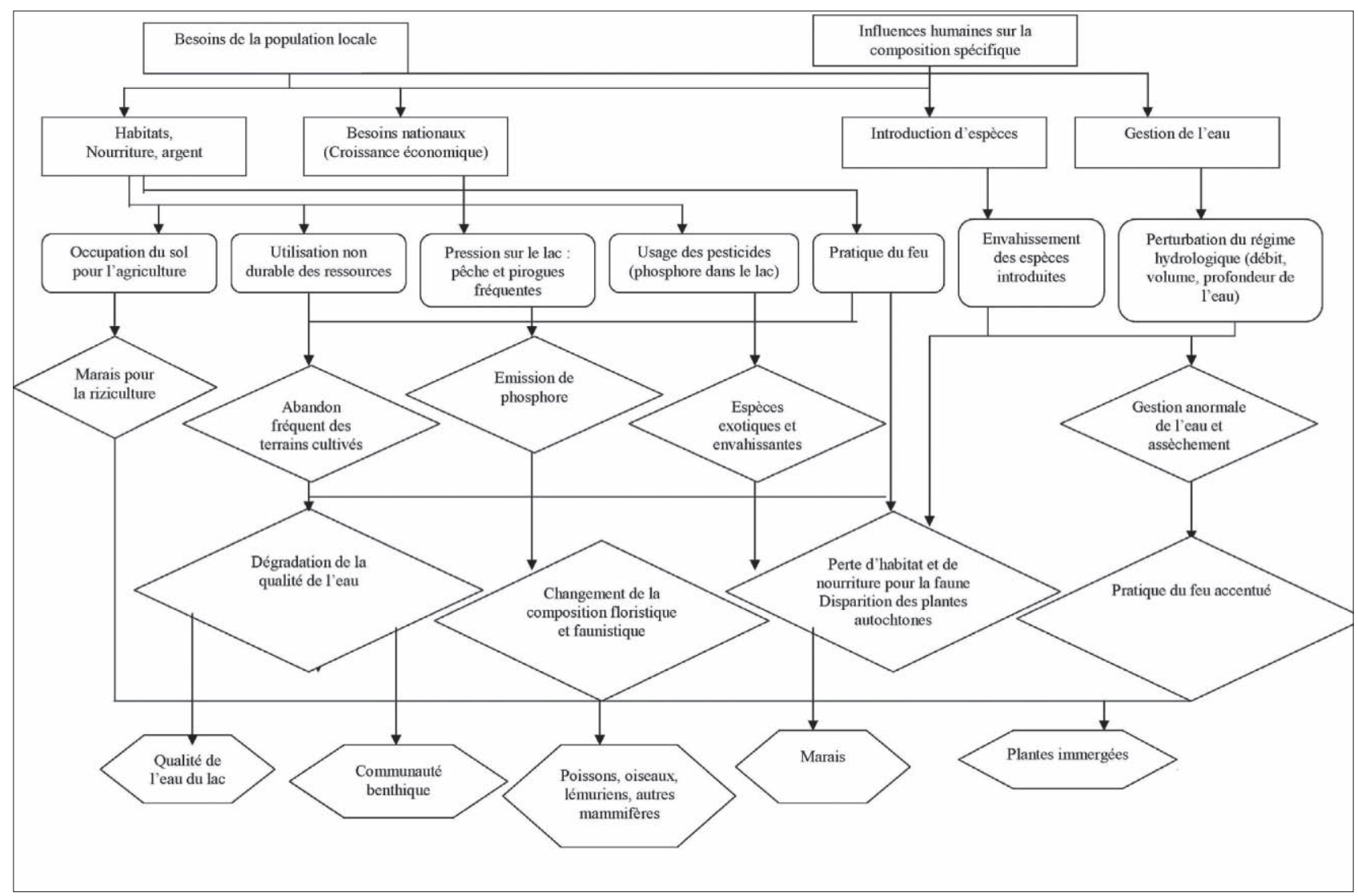

FIGURE 2. Diagramme de concept écologique.

Pressions anthropogéniques;

LES INFLUENCES ANTHROPOGENIQUES ET LES REPONSES ECOLOGIQUE Trois sources majeures de réactions anthropiques ont été identifiées dans la zone humide Alaotra, pour subvenir aux besoins de la population. II s'agit (1) des principaux besoins qui sont l'agriculture, la pisciculture; (2) l'introduction des espèces exotiques telles que les poissons; (3) et la gestion de l'eau.

La faune endémique de l'Alaotra est menacée par le changement de l'environnement: par la dégradation de l'habitat, la surpêche et la chasse excessive; la compétition par introduction des espèces de poissons, la sédimentation due à l'érosion, la pollution humaine par l'utilisation des pesticides et l'invasion des plantes envahissantes (Andrianandrasana et al. 2005).

Agriculture: La zone humide Alaotra est le premier grenier à riz de Madagascar, avec une production annuelle de 250,000 tonnes de riz (Rakotonierana 2004). La population locale dépend de la production rizicole. Pour avoir une meilleure production, divers aménagements ont été effectués par la compagnie Somalac en 1961 (irrigation, élargissement ou diversion des rivières comme la Sahabe, la Sahamaloto, I'Anony, le canal de Mahakary et d'Ampilahoana). Ces aménagements entraînent une diminution de la superficie de l'habitat naturel de la faune (Figure 3). L'emploi des divers engrais et pesticides entraîne un changement de la composition physico-chimique de l'eau et cause un impact négatif sur la présence des espèces aussi bien floristique que faunistique.

La déforestation des bassins versants entraîne l'érosion qui accélère l'ensablement des canaux d'irrigation. Par conséquent, une diminution presque de moitié de la produc- tion rizicole a été constatée. Les villageois ne cessent de réclamer de nouvelles aires du marais intact pour l'agriculture, entraînant la formation de mosaïque de végétation aquatique (Figure 3). La surface de marais brûlée varie de 7,300 ha (31,7\%) à 2,500 ha (> 10\%) en 2003 pour être transformée en rizières (Andrianandrasana et al. 2005).

Changement du régime hydrologique: La dégradation du système d'aménagements pèse sur la zone humide Alaotra, ce qui entraîne un changement dans le temps et dans l'espace du régime hydrologique (débit, volume, profondeur de l'eau) (Moreau 1987). L'eau du lac et du marais est mal gérée à cause de la sédimentation. Des rizières (ancien marais) ont été abandonnées à cause du manque d'eau ou d'une inondation excessive; d'autres endroits du marais avec de l'eau ou mieux irrigués sont brûlés pour être transformées en rizières. Une grande superficie de rizières a été détruite. Le problème en eau devient de plus en plus sérieux; une partie du marais est asséchée et abandonnée (Figure 3). La gestion de l'eau entraîne un changement du milieu naturel tant au niveau de la profondeur de l'eau, qu'au niveau de la biologie et l'écologie des attributs de la zone humide (Ogden et al. 2005).

Influence de la pêche: Le lac Alaotra est une source exclusive pour la population. En 1963, environ 1,000 pêcheurs ont été identifiés (Kiener 1963). Ils utilisaient un système de barrages fait avec de la boue ou servent des hameçons ainsi que d'autres matériaux pour capturer les poissons. A cette époque, plus de 150 pêcheurs ont servi de filets et plus de 30,000 pêches en ligne ont été recensées (Un pêcheur peut avoir plusieurs matériels de pêche en ligne). D'autres pêcheurs ont utilisé du "harato», qui est un système de panier qu'on laisse traîner ou 
déposer dans des endroits choisis, pendant un certain temps voulu pour piéger les poissons. La production annuelle en poissons décline de 4,000 tonnes (en 1960) à 2,000 tonnes en 2004 (Razanadrakoto 2004). Le nombre de pêcheurs augmente de 1,000 à 4,000 en 2003 (Razanadrakoto 2004). La consommation de poissons est de $5.33 \mathrm{~kg}$ par personne en 2001 dans les cinq sites suivis par Durrell Wildlife à $7.21 \mathrm{~kg}$ par personne dans les 16 sites en 2003 (Andrianandrasana et al. 2005).

Actuellement, I'affluence accentuée a été toujours constatée dans la zone humide Alaotra. Le nombre de la population de la région Alaotra augmente de 109,000 (Pigeon 1996) à 550,000 à ce jour (PRD 2003, selon Andrianandrasana 2005). Cette augmentation a une influence sur l'habitat naturel de la faune dans le lac et le marais (la mise à feu, l'aménagement) mais aussi sur la faune abritée dans cette zone humide (la surpêche, la disparition des poissons autochtones).

Pratique du feu pendant la saison sèche: Le feu est périodique dans le marais, quand le niveau de l'eau baisse et que le milieu s'assèche. La mise à feu est pratiquée pour chasser les lémuriens et les poissons Ophiocephalus striatus, mais également pour l'agriculture afin d'augmenter la fertilité du sol selon la population locale. Depuis 2001, les feux sont également pratiqués en vue de créer des étangs artificiels pour la pêche car près de $70 \%$ des étangs naturels dans le marais sont envahis par les espèces exotiques (Andrianandrasana et al. 2005).

Influence des pesticides sur la qualité de l'eau: L'usage des pesticides est libre dans la région Alaotra. Dans le lac, une forte teneur en phosphore et faible teneur en nitrogène ont été détectées (Pidgeon 1996). 34 sortes de pesticides sont en vente dans la région (Pidgeon 1996). Entraînés par l'eau, les éléments tels que le phosphore et le nitrogène peuvent causer une prolifération des phytoplanctons ainsi qu'une végétation en macrophytes rabougrie. Ce qui présente un impact sur la faune qu'il abrite.

Influence de l'homme sur la composition spécifique:

Flore: Le feu induit une répartition en mosaïque de la végétation. En plus, celui-ci entraîne un effet néfaste en dévastant certaines zones du marais (Figure 3). Ce qui favorise I'installation des espèces exotiques.

Faune: Depuis 1926, plus de dix poissons ont été introduits accidentellement ou volontairement par la Direction des Eaux et Forêts, afin d'améliorer la qualité et quantité nutritionnelle dans le lac Alaotra (Kiener 1962, Moreau 1979), mais également pour contrôler et éradiquer les moustiques (introduction volontaire de Gambusia holdrooki en 1940 ainsi que Ophiocephalus striatus en 1980 pour la pisciculture).

Lachasse entraîne la disparition de deuxoiseauxendémiques qui sont Aythya innotata et Tachybaptus rufolavatus (Mutschler 2003). Selon Andrianandrasana et al. (2005), 5,600 oiseaux ont été chassés en 2003 selon les enquêtes effectuées par Durrell Wildlife dans 16 villages aux alentours de la zone humide Alaotra. Ce nombre a diminué de 4,800 en 2002. Les oiseaux résidents dans la zone humide sont chassés. Citons Dendrocygna viduata, Sarkidiornis melanotos, Anas melleri. Trois lémuriens ont été chassés en 2003 s'ils étaient quatre en 2002.

Envahissement des espèces exotiques:

Flore: Les principales espèces exotiques envahissantes de la zone humide Alaotra sont la jacinthe d'eau Eichhornia crassipes et la fougère d'eau Salvinia hastata. Leur installation est favorisée par la dégradation de la qualité de l'eau du lac.

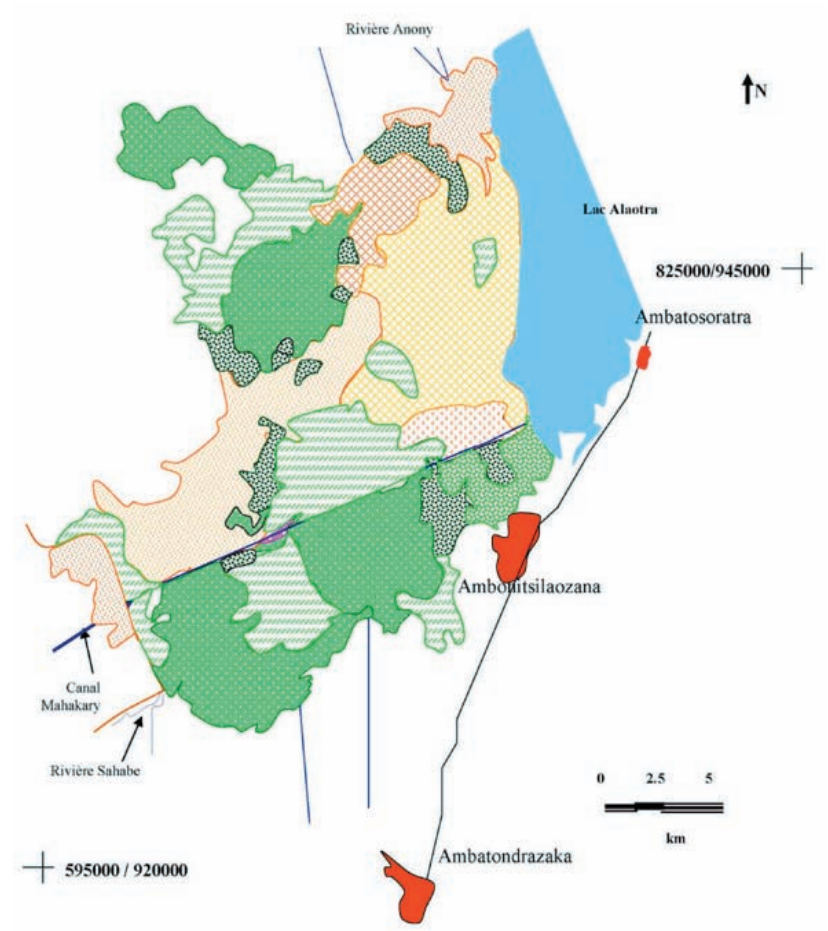

FIGURE 3. Fragmentation du marais Alaotra (carte de végétation établie en Juillet-Août 1997)

Cyperus latifolius associée à des espèces herbacées

Cyperus madagascariensis

Zone dégradée avec des îlots de Cyperus madagascariensis

Typhonodorum lindleyanum ou T. lindleyanum associée à Cyperus madagascariensis

Z Zone dégradée avec Cyperus madagascariensis associée aux Phragmites, des espèces herbacées et des espèces flottantes telles que Eichhornia crassipes, Salvinia hastata

Zone dégradée avec Cyperus madagascariensis à espèces herbacées

$\square$ Rizières abandonnées avec des îlots de cyperus madagascariensis et Phragmites

Phragmites mauritianus

Faune: Les poissons endémiques sus-cités sont devenus rares à cause de l'introduction des espèces exotiques, qui sont majoritaires dans le lac. II s'agit de Osphronemus goramy, Carassius auratus (introduits en 1900), Cyprinus carpio (en 1926), Salmo indicus, S. fario; les Cychlidae tels que Tilapia melanopleura (en 1955), T. rendalli (en 1955), T. zillii (en 1955). II y a aussi d'autres espèces introduites tels que Oreochromis macrochir et O. mossambicus (en 1960), O. striatus (en 1980), qui possèdent une importante biomasse dans le lac Alaotra (Pidgeon 1996).

IMPACTS ECOLOGIQUES Les impacts induits par dif-

férents facteurs dans la zone humide Alaotra sont: la fragmentation de I'habitat (Figure 3), le changement du régime hydrologique, la dégradation de la qualité de l'eau, la dévastation de certaines zones par le feu, la disparition des espèces, de I'habitat et de la nourriture de la faune autochtone, la disparition des ressources naturelles de la population.

Fragmentation de l'écosystème: Les espèces telles que Salvinia hastata et Eichhornia crassipes colonisent la surface libre de l'eau après la mise à feu. Ce qui entraîne un changement tangible de I'habitat. D'autres espèces végétales envahissantes deviennent très communes et colonisent I'habitat (Andrianan- 
drasana 2002). Elles sont associées à la principale espèce qui compose la végétation climacique. Les espèces herbacées sont surtout caractérisées par Polygonum tomentosum, Polygonum wellensi, Salvinia hastata, Eichhornia crassipes, et Aeschynomene elaphroxylon. Ce changement de I'habitat naturel entraîne une forte perturbation de la vie de la faune qui colonise le milieu auparavant. Des espèces de lémuriens se déplacent pour trouver I'habitat approprié. Dans le lac, le peuplement de Phragmites mauritianus se disperse en îlots ou alignés. La répartition en îlots est due, d'une part à la dispersion des graines de Phragmites par les oiseaux Acrocephalus et d'autre part à l'usage des fragments de leur tige pour piéger les poissons, dans les zones temporairement inondées ou non. Ces fragments peuvent être transportés par l'eau et le vent, ils poussent quand les conditions leurs sont favorables. Les fragments utilisés comme piquets pour le piégeage des poissons repoussent et se répartissent de façon alignée (Ranarijaona 1995).

Dégradation de la qualité de l'eau: Une quantité importante de sable et de sédiments envahit les rizières, les terrains de culture ainsi que les canaux d'irrigation et le fond du lac, à cause de la sédimentation. La transparence de l'eau du lac est faible (0.50 m) (Ranarijaona 1995). Un symptôme d'eutrophisation serait probable pour le lac Alaotra si la sédimentation continue. L'utilisation des herbicides ou pesticides entraîne la pollution de l'eau. Ce qui favorise l'installation des plantes envahissantes telles que Eichhornia crassipes et Salvinia hastata, entraînant la disparition de la faune qui y abrite. Une supposition a été évoquée que le changement de la composition chimique de l'eau du lac aurait pu provoquer la mort de quelques poissons trouvés flottés à la surface de l'eau (Pidgeon 1996). Les engrais chimiques utilisés pour l'agriculture affectent l'eau du lac ainsi que la faune et la flore. Certaines hydrophytes sont sensibles aux herbicides. La disparition des hydrophytes pose un problème à la nourriture aux poissons. Ainsi, l'usage des pesticides aux alentours du lac Alaotra entraîne une dégradation de la composition chimique de l'eau et favorise le développement des espèces envahissantes qui rendent asphyxiées la faune.

Disparition de la flore autochtone, habitat et nourriture: L'installation des espèces introduites telles que Polygonum wellensi, Cyperus prolifer, Echinochloa crusgalli, Aeschynomene elaphroxylon, Leersia hexandra, Ludwigia leptocarpa est favorisée à cause de la dégradation de l'écosystème. Ce qui provoque la formation de mosaïque de marais. Sachant que 39 espèces de plantes aquatiques servent de nourriture pour les oiseaux appartenant au tribu des Aythyini (Pidgeon 1996), elles risquent de disparaître à cause de la dégradation de la qualité de l'eau, de la sédimentation ainsi que l'envahissement des espèces exotiques. L'installation des espèces introduites peuvent également induire un changement de la composition floristique du lac par compétition interspécifique, ainsi qu'une disparition des espèces immergées telles que Potamogeton octandrus, Utricularia stellaris, ceratophyllum demersum. Ce qui entraîne un déséquilibre dans l'écosystème.

Disparition de la faune autochtone: La faune telle que Hapalemur alaotrensis ainsi que d'autres mammifères et oiseaux sont menacés par la dégradation de leur habitat et de la disparition de leur nourriture. Deux espèces endémiques d'oiseaux sont actuellement en extinction à cause de l'influence humaine. II s'agit de Aythya innotata et Tachybaptus rufolavatus (Mutschler et al. 1995). Pendant les cinq dernières années, la population des lémuriens a été déclinée de $30 \%$; si la population d'Hapalemur alaotrensis était de 10,000 en 1995, ce nombre diminue de 1,000 à 3,000 lémuriens par an (Mutschler et al. 2001). Les espèces de poissons autochtones telles que Paratilapia polleni sont disparues par l'introduction de Gambusia holbrooki (Mutschler 2003). Les poissons autochtones telles que Rheocles ne représentent que seulement $0.27 \%$ de la population (Pidgeon 1996). Les espèces benthiques qui servent de nourriture pour les poissons et les oiseaux sont disparues également à cause de la sédimentation. Les espèces endémiques telles que Anguilla mosambica et Anguilla marmorata se sont déplacées et devenues rares à cause de la pression humaine.

\section{CONCLUSION}

Cet article essaie d'examiner et de rassembler toutes les recherches effectuées sur la zone humide Alaotra, ainsi que la végétation du marais Alaotra et l'impact de la dégradation sur la faune et la flore. Ces différentes recherches n'ont qu'un seul objectif commun: gestion durable et conservation. Ces résultats de recherche ne sont pas exhaustives, mais peuvent résumer à quel degré d'importance possède la zone humide Alaotra; ils permettent de justifier le classement de la zone humide Alaotra dans le site Ramsar et en Nouvelle Aire protégée en Janvier 2007. Elle est très particulière par sa richesse en faune endémique unique au monde mais aussi par ses ressources naturelles. De nombreuses vies dépendent de cette zone humide aussi bien sur le plan écologique que socioéconomique. Cependant, des influences anthropogéniques ont été constatées dans cette zone humide, malgré les différentes sensibilisations et éducations environnementales offertes aux villageois. La superficie du marais ne cesse de diminuer par la mise à feu pour le transformer en rizières. La végétation climacique du marais est détruite ou dégradée, la surface libre laissée par la mise à feu est recolonisée par des espèces flottantes envahissantes ou du Phragmites. Des rizières issues de I'ancien marais sont abandonnées à cause du problème en eau. Cependant, la sensibilisation des villageois est fructueuse car une régénération de la végétation du marais est constatée et que les villageois sont conscients de la gestion durable et de la conservation du marais.

Le présent travail offre le concept de modèle écologique, en décrivant I'interdépendance entre les différentes entités écologiques. Ce modèle pourrait persuader les autorités locales ainsi que les villageois d'élaborer ensemble avec les chercheurs un programme de restauration dans le futur. Dans tout les cas, le reboisement aux alentours de la région Alaotra, la conservation durable des forêts existantes, ainsi que I'application des lois dans la conservation de la zone humide Alaotra sont les clés pour sa restauration, afin d'aboutir à une meilleure conservation durable.

\section{SUGGESTIONS DE RECHERCHE}

La Figure 3 nous a permis de savoir l'état de dégradation du marais 10 ans passés. Si on fait une comparaison avec la carte du marais «occupation des sols site Ramsar» en 2005 (Figure 1), une évolution promettant est remarquée au niveau de la régénération de la végétation du marais. Ce qui est également constatée en regardant une diminution tangible de la superficie des marais brûlée de I'an 2000 (de l'ordre de 7,000 ha) à 2003 (sur une superficie de 2,500 ha (Andrianandrasana et al. 2005). 
Celle-ci est sans doute due à la sensibilisation des villageois faite par Durrell Wildlife ainsi que les différentes entités. Les villageois ont apprécié la sensibilisation malgré les différentes lois imposées pour la gestion durable et la conservation de la zone humide Alaotra. Cette sensibilisation a créé un contact ainsi qu'une collaboration entre les villageois et le gouvernement (Andrianandrasana et al. 2005). C'est bien I'un des objectifs du programme du gouvernement actuel dans le MAP (Madagascar Action Plan): «fampandrosoana miainga avy eny amin'ny vahoaka ifotony ». Cette dégradation n'aurait cessé de s'accentuer si aucune mesure n'avait été prise pour la conservation de la zone humide Alaotra. C'est la raison pour laquelle I'objectif concernant la conservation de la zone humide Alaotra en site Ramsar et en Nouvelle Aire protégée est atteint. II se trouve d'après les cartes site Ramsar qu'une grande partie du marais est devenue 'zone sensible', un site de conservation de la faune menacée existe déjà, un site de patrimoine culturel est identifié dans la zone humide Alaotra; pour les préserver, I'application des règlements communautaires agréés par tous les villageois ou 'dina', est une des issues pour contrôler la pêche et maintenir le marais (Durbin 2003).

Des recherches multidisciplinaires seront encore nécessaires pour conserver et mieux gérer la ressource de manière durable. Voici quelques suggestions de recherche: Pour convaincre le monde concerné à la zone humide Alaotra, il est nécessaire de faire connaître l'existence du présent concept de modèle écologique. Des recherches méritent d'être lancées:

- Flore: Etude de l'état actuel du marais par photosatellite la plus récente; écologie et dynamisme de croissance de marais dans le but de sa restauration; la diversité et l'écologie des phytoplanctons du lac Alaotra afin de confirmer l'état du lac s'il y a eutrophisation ou non.

- Faune: Etude des impacts majeurs de l'absence des deux oiseaux endémiques en extinction; essai de multiplication des principales espèces survivantes.

- Hydrologie: Analyse du régime hydrologique et de la qualité de l'eau du lac, causés par les pressions anthropogéniques. Etude de la qualité de l'eau des rivières et du lac à l'aide des macrophytes, par l'utilisation de I'Indice Biologique des Macrophytes des Rivières (IBMR).

- Agriculture: Approche participative: persuader la population locale que "sans marais = sans lac = sans vie»; sensibilisation de la population locale d'appliquer des techniques modernes (usage des variétés améliorées, respect de calendrier cultural) pour la production rizicole ainsi que d'autres cultures pour éviter la réclamation sans cesse du marais et le problème d'épuisement du sol.

Conservation: Le classement de la zone humide Alaotra dans le site Ramsar et en nouvelle aire protégée est une décision incontournable et très méritante, vu sa richesse en faune et sa flore d'une part et les ressources naturelles et les différents intérêts qu'elle apporte à la population locale et nationale d'autre part. La création de nouvelles aires protégées pour certaines zones cibles dans les bassins versants Alaotra est une clé pour lutter contre l'érosion. Cette érosion a une influence néfaste sur la zone humide Alaotra.

La réalisation d'une étude d'impact environnemental et socio-économique suite à la création de la Nouvelle Aire Protégée est vivement souhaitable.

\section{REMERCIEMENTS}

Je tiens à remercier très chaleureusement $\mathrm{Dr}$. Jonah Ratsimbazafy pour ses précieuses collaborations à l'élaboration du concept de modèle écologique de la zone humide Alaotra. Mes reconnaissances s'adressent à Dr. Ferry L. (IRD) et Dr. Robison L. (CNRE) ainsi que toute l'équipe de I'IRD, à I'IRD et au BRL de m'avoir financée et m'aidée à la réalisation des différents travaux de terrain. Je réitère mes remerciements à Dr. Razafimandimbison S. pour la traduction en anglais du résumé.

\section{REFERENCES BIBLIOGRAPHIQUES}

Aldegheri, M. 1972. Rivers and streams on Madagascar. In: Battistini R. \& G Richard-Vindard. 1972. Biogeography and ecology in Madagascar, pp 261-267. Dr. W. Junk B.V., The Hague.

Andrianandrasana T.H. 2002. Etude de la régénération du marais Alaotra, habitat naturel du Hapalemur griseus alaotrensis. Durrell Wildlife Conservation Trust - Madagascar Programme. Antananarivo, Madagascar.

Andrianandrasana, H.T., Randriamahefasoa, J., Durbin, J., Lewis, R.E., and Ratsimbazafy, J.H. 2005. Participatory ecological monitoring of the Alaotra wetlands in Madagascar. Biodiversity and Conservation Journal, 14:2757-2774.

Durbin, J., Rakotoniaina, L.J., Randriamahefasoa, J. 2003. Project Alaotra: using endangered species as flagships for community based wetland conservation. In: The natural history of Madagascar, M.Goodman and J.P. Benstead (eds.), pp. 1551-1555. The university of Chicago press, Chicago and London.

Ferry, L., Robison, L., Ranarijaona, H., Gasse, F. 1999. Les lacs de Madagascar: présentation et typologie. IRD, Montpellier (unpublished).

Ogden, J.C., Davis, S.M., Kimberly, J., Barnes, J.T., Fling. H.E. 2005. The use of conceptual Ecologicals Models to Guide Ecosystem Restoration in South Florida. Journal of the Society of Wetland Scientists, USA, 25, 4:795-809.

Kiener, A. 1962. Evolution de la pisciculture et de la pêche dans les eaux intérieures de Madagascar depuis 1950. Bulletin de Madagascar. 1033-1044.

Kiener, A. 1963. Poissons, pêche et pisciculture à Madagascar. Centre technique Forestier Tropical, 24, Nogent sur Marne. 404.

Langrand, O. 1995. Guide des oiseaux de Madagascar. Delachaux et Niestlé, Lausanne, Switzerland. In: Participatory ecological monitoring of the Alaotra wetlands in Madagascar Andrianandrasana, H.T., Randriamahefasoa, J., Durbin, J., Lewis, R.E., and Ratsimbazafy, J.H. (2005). Biodiversity and Conservation Journal, 14:2757-2774.

Minenvef, WWF, Conservation International, Durell Wildlife Conservation Trust 2005. Cartes de grandes fonctions, Site Ramsar Alaotra 722.500 ha.

Moreau, J. 1987. Madagascar. In: M. Burgis and J.J. Symoens (eds) African wetlands and shallow water bodies, pp 595-606. Orstom, Paris.

Mutschler, T., Feistner, A.T.C. 1995. Conservation status and distribution of the Alaotran gentle lemur Hapalemur alaotrensis. Oryx, 29: 267-274.

Mutschler, T. Nievergelt, C., Feistner, A.T.C. 1995. Human induced loss of habitat at lac Alaotra and its effects on the Alaotran gentle lemur. In: Environmental change in Madagascar, Petterson B.D., S.M., Goodman, J.L., Sedlock (eds.), pp 35-36. Field Museum, Chicago.

Mutschler, T., Randrianarisoa, A.J., Feistner, T.C. 2001. Population status of the Alaotran gentle lemur Hapalemur alaotrensis. Oryx, 35:152-57.

Mutschler, T. 2003. Lac Alaotra. In: The natural history of Madagascar, S.M. Goodman, J.P., Benstead (eds.), pp 1530-1534. The university of Chicago press, Chicago and London.

Odgen, C.J. 2005. Everglades ridge and slough conceptual ecological model. Journal of the society of Wetland Scientists, USA, 25, 4:810-820.

Pidgeon, M. 1996. An ecological survey of lake Alaotra and selected wetlands of central and eastern Madagascar in analysing the demise of Madagascar pochard Aythya innotata. Lucienne Wilmé edition, WWF, Antananarivo.

Rakotonierana. D. 2004. Rapport Annuel 2003 CIRAGRI. Circonscription d'Agriculture. Ambatondrazaka, Madagascar. 
Ranarijaona, H.L.T. 1994. Méthode d'approche pour l'étude de la végétation aquatique de Madagascar. Rapport LRSAE Hydrobiol (unpublished).

Ranarijaona, H.L.T. 1995. Etude préliminaire de la végétation aquatique du lac Alaotra. Rapport LRSAE Hydrobiol (unpublished).

Ranarijaona, H.L.T.1998. Etude de la végétation du marais Alaotra. Rapp. LRSAE Hydrobiol (unpublished).

Ranarijaona, H.L.T. 1999. La flore des milieux lentiques malgaches (lacs, marais, étangs): essai de typologie. Unpubl. Doctorat du 3ème cycle. Université d’Antananarivo, Madagascar.
Razafindrakoto, D. 2004. Rapport Annuel 2003 CIRPRH. Circonscription de la Pêche et des Ressources Halieutiques, Ambatondrazaka, Madagascar. In: Participatory ecological monitoring of the Alaotra wetlands in Madagascar. Andrianandrasana, H.T., Randriamahefasoa, J., Durbin, J., Lewis, R.E., and Ratsimbazafy, J.H. (2005). Biodiversity and Conservation Journal, 14:2757-2774. 